\title{
Alcune particolarità della comparazione (quomodo \\ - sic, quemadmodum - sic, ita uti - sic) in latino volgare, con particolare attenzione alle defixiones
}

Daniela Urbanová

(Masaryk University, Brno)

\section{Some Particularities of Comparison (quomodo - sic, quemadmodum - sic, ita uti - sic) in Vulgar Latin with Special Attention to defixiones}

Vorrei dedicare il presente contributo alla memoria del mio caro maestro, professor Antonín Bartoněk, eccellente uomo di scienza e ispiratore di molte ricerche, il quale tempo fa ebbe a richiamare la mia attenzione sulle defixiones latine.

\begin{abstract}
The documentation of Latin curse tablets offers authentic evidence of the use of comparison in Latin. Moreover, it has recently been enriched by new findings at the fountain of Anna Perenna and in Mainz (the Roman Mogontiacum). Regarding Latin epigraphic evidence, curse tablets primarily use comparative clauses introduced by quomodo - sic, quemadmodum - sic, and rarely also ita uti - sic. These are included in the so-called simile formula, which is nowadays well attested from all provinces of the Roman Empire. The complex structures and formularies used in the curse texts are usually interconnected with magical ritual procedures. The simile formula acts through so-called persuasive analogy, the aim of which is to influence future events according to aforethought model. This contribution endeavours and to analyse the phonetic-phonological, functional, and semantic development of the Latin comparative conjunction quomodo with special attention paid to the Vulgar Latin elements included.
\end{abstract}

\section{Keywords}

quomodo - sic; comparison; analogy; Vulgar Latin; apocope; subjunctive; curse tablets 
Inizierò ripercorrendo brevemente le vicende di quomodo e dei suoi sviluppi fonetici, ben riflessi nei testi delle defixiones, e anche di quelli funzionali, avvenuti nell'ambito del latino letterario e soprattutto volgare.

Quomodo costituito dall'aggettivo indefinito qui (relativo o interrogativo) e il sostantivo modus, ambedue in ablativo, formano un avverbio composto (si veda Bodelot 2010: p. 207). In forma non univerbata è attestato in Plauto e appare relativamente di rado nell'epoca preclassica: si vedano Hoffmann \& Szantyr (1965: pp. 649-650), Tarriño (2011: pp. 373-376) e Bodelot (2010: p. 209). La sua funzione interrogativa prevale in Plauto e Terenzio, più frequentemente è documentato in Cicerone e Seneca ma solo raramente in epoca tarda. Il suo uso relativo-comparativo comincia con Plauto e anche lo sviluppo del suo uso con dei correlativi si afferma in epoca classica, si vedano Hoffmann \& Szantyr (1965: p. 649) e Tarriño (2011: pp. 406-407), è documentato in Cicerone e il suo uso si moltiplica nelle opere di Quintiliano e di Seneca filosofo, ormai univerbato introduce le relative-comparative con sic e ita; si veda Bodelot (2010: pp. 210-214). Col suo sinonimo quemadmodum, anch'esso usato già nella lingua parlata di epoca classica, fa concorrenza a ut (Hoffmann \& Szantyr 1965: p. 649 e Herman 2000: p. 91). Quomodo, in una forma ridotta nel latino volgare, diventa in seguito panromanzo: it. arcaico como, poi come; cuemo nello spagnolo, como in portoghese; com, con nel francese e cum in rumeno: si vedano Pirson (1908: p. 65), Väänänen (1982: pp. 273-274).

La riduzione che quomodo subisce nella lingua popolare relativamente presto, per passare poi in tutte le lingue romanze col significato di "come", è ben documentato dalle defixiones. La labiovelare sorda qu è scritta fino al 2. sec. d. C. anche con $c u$, come sostiene Adams (1977: p. 32); ma si vedano anche Kropp (2008a: pp. 264, 261-262), Väänänen (1982: pp. 51-52), Morani (2000: p. 189), Herman (2000: p. 48). ${ }^{1}$

1. $q u \sim c u$

L'alternanza grafica $q u \sim c u$ è attestata ad. es. nelle defixiones di Raetia, Pannonia e Britannia, databile pero dal $2^{\circ}$ sec. d. C. in poi:

(1) Pannonia, Aquincum, Barta 2015 ( $2^{\circ}-3^{\circ}$ sec. d. C.):

... Cuomodo Manes muti et taciti sunt, sic qui tibi antepistulam adferent, muti et taciti sint... ${ }^{2}$

(2) Raetia, Wilten, dfx.7.5./1 (metà del $2^{\circ}$ secolo d. C.):

... deligat, ut persicuatis (=persequatis) et eum aversum a fortunis (s)uis avertatis... ${ }^{3}$

\section{2. comodo}

$q u>c$ : la labiovelare sorda perde la componente labiale dopo la velare e viene notata con la sola C. L'alternanza $q u \sim c$ in quomodo con funzione relativa-comparativa è attestata dai seguenti esempi:

1 Per l'indebolimento di semiconsonante $[\mathrm{w}]$ dopo [k] che porta alle confusioni di grafia $q u \sim Q, C, K \mathrm{e}$ anche $q u \sim C U$ vedi Herman (2000: p. 48).

2 Traduzione inglese Barta (2015: pp. 112-113): "Just as the infernal souls are mute and silent, so those who will bring a curse-in-reply to you may be mute and silent."

3 Per testo completo e traduzione vedi No. (18) più avanti. 
(3) Italia, Pompei, 33.dfx.1.5.4/1 (2 ${ }^{\circ}$ sec. a. C.): ... comodo is eis $\operatorname{desert(us)...~}{ }^{4}$

(4) Germania, Colonia (metà del $1^{\circ}$ sec. d. C): ... comodo hoc perverse scriptu $(m)$ est... ${ }^{5}$

(5) Africa, Cartagine, $117 \mathrm{dfx} .11 .1 .1 / 7\left(2^{\circ}-3^{\circ}\right.$ sec. d. C): ... comodo Securus non potes $(t)$ loqui sic... ${ }^{6}$

(6) Pannonia, Aquincum, Barta 2016 (2 $2^{\circ}-3^{\circ}$ sec. d. C.): ... Ad Tartara tradas comodo epistularius qui tibi epistulas tradet... ${ }^{7}$

Gli esempi della grafia $c$ al posto di $q u$ attestati dalle tavolette nella parola quomodo non sono molto numerosi, ma la loro distribuzione nell'area tra Italia, Germania e Pannonia, Africa e Britannia rispecchia una diffusione in tutto l'impero Romano. Per quanto riguarda la cronologia, si va dal $2^{\circ}$ secolo a. C. in Campania al $1^{\circ}$ sec. d. C. in Germania, mentre tutti gli altri esempi sono databili tra il $2^{\circ}$ e $3^{\circ}$ secolo d. C. Al di fuori dell'ambito delle defixiones, quomodo compare in poche iscrizioni. ${ }^{8}$

\section{3. como}

Subita l'apocope, quomodo passa a como secondo la trafila quōmodo > quōmo >como. Le più antiche occorrenze di questo sviluppo sono attestate proprio dalle defixiones:

(7) Pompei, dfx.1.5.4/12 (2 ${ }^{\circ}$ sec. a. C.):

A: ... P(hi)lematio Hostili (serva) faciam (=faciem) ... capil $(l) u(m)$, cerebru $(m)$, flatus, ren(es) ..., ut illai non suc(c)edat ... ut ilic (=ille) illa $(n) c$ (=illam) odiat. Como(do) ... (h)aec nec agere ne ilai (=illa) ... qui $(c)$ qua $(m)$ agere pos(s)it ... P(hi)lematio ... B: nec agere nec ul(l)a(s) res pos(s)it pete(re), quae ul(l)o (h)uma(no) ... comodo is eis desert(us), ilaec (=illa) deserta sit cu(n)no... ${ }^{9}$

La tavoletta da Pompei, datata al secondo secolo avanti C. viene citata da Väänänen (1982: p. 101) come primo esempio di apocope, ma, vista la seconda occorrenza di comodo non apocopata più avanti nello stesso testo, ciò non si può ritenere del tutto sicuro. Un altro possibile esempio di apocope citato da Pirson (1908: p. 63) e altri, proviene da Bath in Gran Bretagna, ed è datato al 2.- 3. secolo dopo C. Nel testo gli sconosciuti ladri

4 Per testo e traduzione vedi No. (7) più avanti.

5 Vedi Blänsdorf \& Kropp \& Scholz (2010: pp. 272-276): “... just like this is written in a twisted way [the text is written right-to-left, i.e. in an unusual manner]. Le interpretazioni delle tavolette sono basate su Urbanová (2014), tradotte in inglese da N. Gachallová, se non indicato altrimenti.

6 (“... just like Securus cannot speak, so...”.)

7 Vedi Barta (2016) con la traduzione: "As a messenger, hand over to Tartarus those who will hand letters to you."

8 La Clauss-Slaby Epigraphik-Datenbank riporta in tutto 53 iscrizioni latine, che contengono la parola quomodo. Di esse 34 vengono dalle defixiones, mentre nelle iscrizioni comuni, quomodo nel senso relativo-comparativo compare solo poche volte (Didymoi 00429; CLE 01542; AE 2007, 01029; AE 1976, 00094; CLE 01798a; AE 1980, 00051). Le altre iscrizioni non usano quomodo in senso relativo-comparativo in una proposizione complessa, ma piuttosto con significato temporale o equivalente a ut in un comparazione semplice, oppure sono troppo frammentarie.

9 ("Philematio, the slave of Hostilius: [I curse? her] face ... hair, brain, breath, kidneys ..., may she not succeed ... may he hate her. Just like ... this one [can] not do anything ... may she equally be unable to do anything ... Philematio ... may she be unable to act or to ask for anything, what to any human? ... Just like this one is deserted by them, may she be deserted in her bed..."). Deserta sit cunno, more accurately "may she be deserted in her lap". 
di una fibula devono sciogliersi come acqua. Il testo è stato redatto da sinistra a destra nella giusta successione, ma con le lettere di ogni singola parola invertite. Così nella seconda riga si legge dalla fine $A Q U A C O M C$, dove la $C$ potrebbe essere considerato un semplice errore per la $\mathrm{O}$, e quindi per COMO. Il contesto comparativo è assicurato dal SIC che segue.

(8) Britannia, Bath, dfx.3.2/1 (2º $-3^{\circ}$ sec. d. C.): Qu(i) mihi VILBIAM (= fibulam) in(v)olavit, sic liqu(esc?)at com(odo) aqua... ${ }^{10}$

Due esempi epigrafici più sicuri di como corrispondente a quomodo, usato come relativo-comparativo, si trovano in una tavoletta di scoperta recente proveniente da Mogontiacum, databile al 65-130 d. C. (Blänsdorf 2012: p. 1). Il testo è scritto con un layout magico: parzialmente sul bordo della tavoletta, incorniciando la parte centrale della maledizione iscritta normalmente al centro: per questa ragione si vede il nome della vittima, Prima Aemilia, nella prima riga superiore capovolto.

(9) Mogontiaco, dfx.5.1.5/4; DTM 15 (65-130 d. C.):

Prima Aemilia Narcissi agat, quidquid conabitur, quidquid aget, omnia illi inversum sit, amentita surgat, amentita suas res agat. Quidquid surget, omnia interversum surgat. (P)rima Narcissi aga(t): como haec carta nuncquam florescet, sic illa nuncquam quicquam florescat. ${ }^{11}$

Un altro esempio di como attestato dalle tavolette di maledizioni proviene anche dalla Germania, precisamente da Gellep (l'antica Gelduba, vicino a Krefeld), ed è databile alla $2^{\circ}$ metà del primo secolo d. C. Il testo, interpretato nelle edizioni precedenti diversamente, risulta, dopo la nuova lettura di Blänsdorf (2014: pp. 181-186), una defixio, con due occorrenze di como: Como hoc perversum scriptum est, sic illos dei spernent... (testo A) e ripetuto ancora una volta nel testo $\mathrm{C}$, però con grafia errata: Conmo oc perversum est, sic... Anche in questo caso il testo ha un layout magico e la formula di maledizione è scritta in due righe verticali.

Il presupposto quōmo (scritto con la qu) non è attestato nei documenti epigrafici, ma appare nei codici del 4.-5. sec. d. C. che contengono il Nuovo testamento citati da Pirson (1908: p. 62).

\section{4. quomodi}

Un'altra variante di quomodo è quomodi, rintracciabile esclusivamente nei testi di maledizioni, attestato in Gallia insieme a quomodo al $2^{\circ}$ sec. d. C. e circa quattro volte in Germania, e anche in Africa dal $2^{\circ} / 3^{\circ}$ sec. d. C. La forma quomodi non è attestata altrove ed è formata forse secondo cuiusmodi, come sostengono Hoffmann \& Szantyr (1965:

10 Vedi il testo e traduzione di Tomlin (1988: No. 4): "May he who has stolen VILBIA [the brooch] from me become as liquid as water ... who has stolen it... [a list of potential culprits follows]." facsimile RIB 154, accessibile http://romaninscriptionsofbritain.org/inscriptions/154.

11 ("[Whatever] Aemilia Prima, [the lover?] of Narcissus may do, whatever she attempts, whatever she does, let it all go wrong [lit. may it be perverted]. May she get up [out of bed] out of her senses/mind, may she go about her work out of her senses/mind. Whatever she strives after, may her striving in all things be reversed. May this befall Prima [the lover?] of Narcissus: just as this tablet shall never bloom, so she shall never bloom in any way.”) 
pp. 649-650); Pirson (1908: p. 64) suggerisce invece un rifacimento secondo il modello sicuti, veluti, ma questa ipotesi appare meno probabile. Citiamo qui alcuni esempi: Germania, Mogontiacum DTM 6 (65-130 d. C): Q(u)omodi et ille mihi fraudem fecit, sic illi...; Gallia, Chagnon, dfx.4.3.1/1 (metà del $2^{\circ}$ sec. d. C): Quomodi nec mater huius catelli defendere potuit... sic nec advocati eorom e( os d)efendere possint...; ${ }^{12}$ Africa, Cartagine, dfx.11.1.1/8 ( $2^{\circ}-3^{\circ}$ sec. d. C): Quomodi haec nomina a(d inferos dedi sic omnes)(adversu)s me ommutes(cant) (neque lo)qui (possint?)... ${ }^{13}$

\section{5. comdi}

La forma di quomodo sincopata (Pirson: p. 1908) occorre in un caso solo, ma sicuro, nelle defixiones in una tavoletta proveniente da Keruznach, in Germania, datata alla $2^{\circ}$ metà del $1^{\circ}$ secolo d. C.

(10) Bad Kreuznach, dfx.5.1.4/5 (2a metà del $1^{\circ}$ sec. d. C):

Sinto Vale(n)tis sive alii inimici. Sinto Valentinus inim(i)cus. Sic comdi plumbum subsidet, sic Sintonem et Martialem Sinto(nis) et adiutorium Sintonis et quisquis contra Rubrium fr(atre)m et me Quartionem, si qui(s) contravenerit, Sintonem et adiutorium eius Sintonis defero ad infero $(s) . .{ }^{14}$

Altre peculiarità riguardanti le varianti di quomodo nelle maledizioni, provenienti soprattutto dalla Germania, dimostrano probabilmente una certa insicurezza degli scriventi a proposito delle particelle di comparazione quomodo e anche quemadmodum, attestato solo poche volte, nel primo e secondo secolo dopo C. (si veda Blänsdorf (2012: p. 27): qu(omo)di, quamodum (Mogontiacum, DTM 12); quatmodum (Mogontiacum, DTM 11); quommodi Mautern (dfx.6.1/1). ${ }^{15}$

Ma dobbiamo anche osservare che le già citate varianti di quomodo, così come como, comdi, quomodi, costituiscono un po'meno della metà delle attestazioni di quomodo, mentre nel resto dei testi quomodo appare nella sua forma classica anche nel $4^{\circ} / 5^{\circ}$ secolo d. C.; si veda la dfx.1.4.4/13 da Roma.

Forse può colpire l'occorrenza relativamente alta di quomodo in un corpus epigrafico limitato, se si pensa al corpus letterario molto più ampio. D’altra parte si può dire che nei testi delle maledizioni quasi non compaiono congiunzioni d'uso comune, e diffusissime nei testi letterari. Questo vale soprattutto per le congiunzioni temporali come ad es. cum, postquam e anche causali quod, quia, quoniam. Invece ben attestate sono le congiunzioni finali, consecutive e condizionali, soprattutto ut, ne, ut non e si, nisi (si veda Kropp 2008a: p. 281).

12 Per testo e traduzione vedi No. (17) più avanti.

13 Per testo e traduzione vedi No. (12) più avanti.

14 ("Sinto, [the freedman?] of Valens, or other enemies. Sinto Valentinus [is] an enemy. Just as the lead sinks [to the bottom], so I drive down to the gods of the Underworld Sinto and Martialis, [the son/freedman] of Sinto, and his assistant and whomever, [who is] against my brother Rubrius and me, Quartio, if anyone comes out against [us], Sinto and the assistant of this Sinto...”.)

15 Per le varie grafie volgari di quomodo si deve menzionare anche il progetto guidato da B. Adamik: Computerized Historical Linguistic Database of Latin Inscriptions of the Imperial Age [http://lldb.elte.hu/]. 
Nei testi letterari quomodo è documentato con varie funzioni: nel latino classico nelle interrogative e relative-comparative, di rado anche con valore finale o restrittivo: si vedano Hoffmann \& Szantyr (1965: pp. 649-650), Tarriño (2011: p. 406). Il successivo sviluppo di quomodo nel periodo postclassico dimostra una sua espansione a scapito di ut e velut. Quomodo poteva assumere il significato temporale, causale, quello di quod, quia che sostituiva l'accusativo con l'infinitivo, si vedano Hoffmann \& Szantyr (1965: p. 650), Väänänen (1982: pp. 273-274), Herman (2000: p. 91). Questo uso compare anche nelle epigrafi, ad. es. nelle tavolette di Vindolanda. Nei testi di maledizione quomodo viene impiegato esclusivamente nelle relative-comparative o nelle equative. Tutto ciò è dato dal fatto che i testi delle defixiones sono ripetitivi, mostrano particolari formulari connessi alle procedure magiche, e in certa misura sono anche testi tecnici ed artificiali (vedi Urbanová \& Cuzzolin, 2016: p. 341).

Nei testi delle defixiones troviamo spesso questo tipo di completive, impiegate a formare la simile-formula denominata anche "analogy within request formula", si veda Kropp (2010: pp. 370ff.) che adopera l'analogia persuasiva. L'analogia persuasiva, a differenza di quella empirica, non prevede avvenimenti futuri sulla base di osservazione di identici processi svoltisi nel passato, ma cerca di influenzare gli avvenimenti futuri secondo un modello dato precedentemente. Nel rituale della maledizione si presuppone che, grazie all'analogia persuasiva, si raggiunga un trasferimento delle qualità desiderate (tra due oggetti che hanno qualità diverse) dall'uno all'altro, si vedano Faraone (1991: p. 8), Kropp (2008a: pp. 175-177).

La simile-formula è attestata esclusivamente nei documenti epigrafici, il corpus di 39 testi contiene soprattutto defixiones, mentre si trovano poche occorrenze anche nelle epigrafi non magiche (6 istanze in EDCS, alcune frammentarie, di cui due istanze in CLE 1798 e 1542). La simile-formula è documentata 51 volte in 33 testi di defixiones (31 volte in 22 testi delle maledizioni e 20 volte negli 11 delle preghiere per la giustizia). È attestata in Africa 8 volte, in Italia 10 volte, mentre il massimo delle occorrenze, cioè 20 formule, proviene dalla Germania, 5 volte dalla Gallia e 4 volte dalla Pannonia.

La datazione della simile-formula coll'uso di quomodo nel senso relativo-comparativo-equativo va dal secondo secolo avanti Cristo in Italia, mentre altri due esempi sono ascrivibili uno, provenienti da Roma, al primo sec. a. C., l'altro, proveniente dalla Germania, al primo e secondo secolo dopo C. Altri esempi dalle regioni Danubiane, Africa e Britannia appartengono al secondo o terzo sec. d. C. Un unico testo da Roma è databile al $4^{\circ}-5^{\circ}$ sec. d. C. Questa formula si trova spesso nei testi più lunghi, complessi ed elaborati, come il seguente esempio da Mogontiacum.

(11) Germania, Mogontiacum, DTM 6 (65-130 d. C.):

Quintum in hac tabula depon(o) aversum se suisque rationibus vitaeque male consumantem. Ita uti galli, Bellonarive absciderunt, concideruntve se, $\underline{\text { sic }}$ illi abscissa sit fides, fama, faculit(a)s. Nec illi in numero hominum sunt, neque ille sit. Q(u)omodi et ille mihi fraudem fecit, sic illi, sancta Mater Magn(a), et relegis(ti) cu(n)cta. ${ }^{16}$

16 ("On this tablet I curse Quintus, may the gods avert from him and his business, may he spend [his time] miserably. Just like the priests of Mater Magna [i.e. galli] and the priests of Bellona have castrated or cut themselves, so may his good name, reputation, the ability to conduct his affairs be cut away. Neither they 


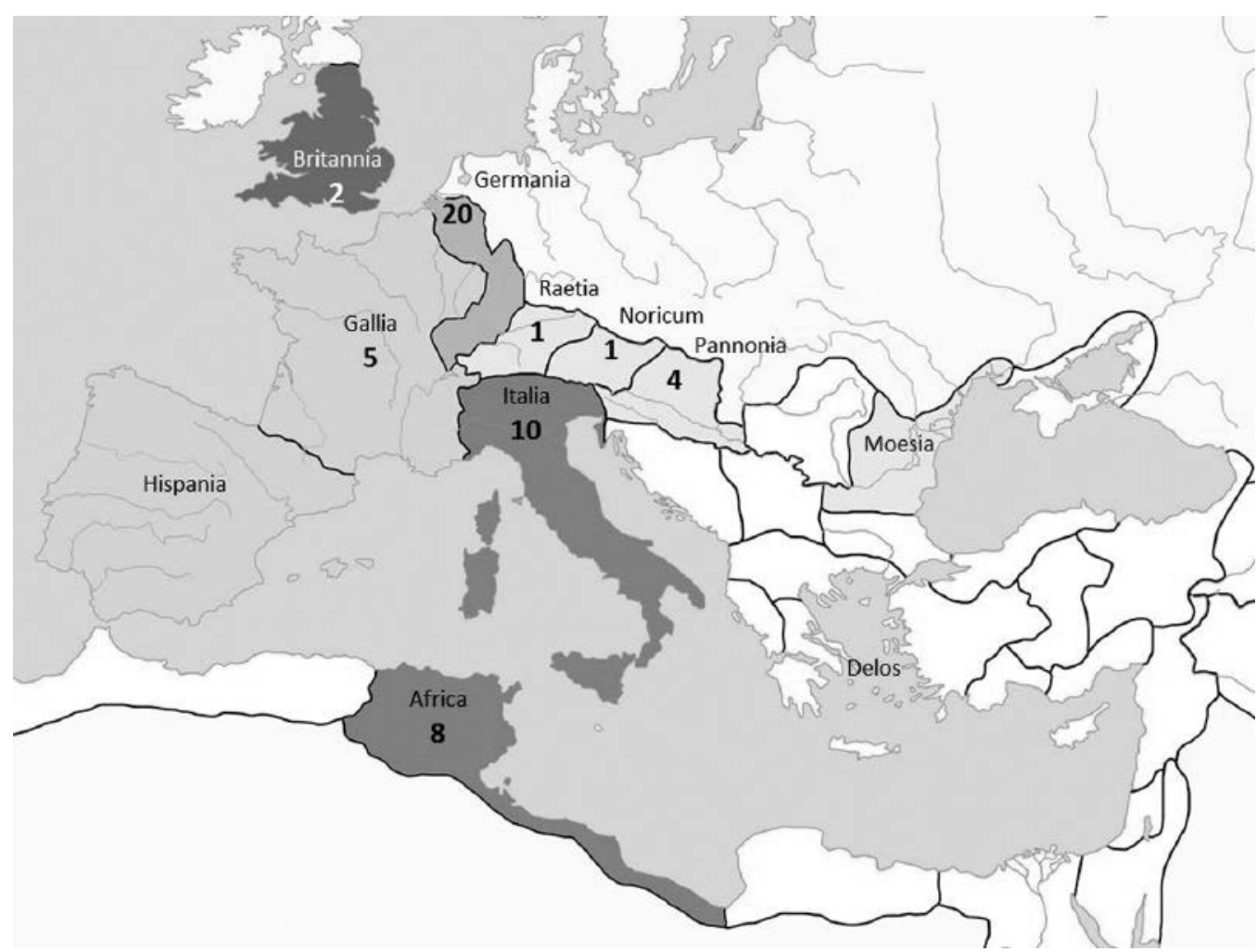

Figure 1: Distribuzione della simile-formula nell'Impero Romano

Questa formula è attestata anche nelle varie situazioni delle maledizioni, spesso ricorre nei testi riguardanti i processi, come vediamo nella tavoletta dell'Africa, dove i nemici, nominati sopra devono essere muti: il paragone crudele è fatto col gallo al quale è stata tolta la lingua.

(12) Africa, Cartagine, dfx.11.1.1/7 (2。-3 sec. d. C.):

Claudia Helene, Clodia Successi, Clodia Steretia, Clodius Fortunatus, Clodius Romanus, Mu(rc)ius ... Servilius Faustus, Valerius Extricatus. Quomodi haec nomina a(d inferos dedi sic omnes) (adversu)s me ommutes(cant) (neque lo)qui (possint?). $(Q u o m o d o)$... huic gallo ... lingua $(m)$ vivo extorsi et defixi, sic inimicorum meorum linguas adversus me ommutescant... ${ }^{17}$

are numbered among mankind, nor may he be. Just like he deceived me, so may [you,] holy Mater Magna take everything away from him.") Per testo e interpretazione vedi Blänsdorf (2012: No. 6).

17 ("[The names of the accursed persons, probably freedmen and freedwomen] Just like [I commended to the infernal gods] these names, [may all] be struck dumb toward me and [may they be unable to speak]. [Just like] I ripped out and transfixed alive the tongue of this cock, may the tongues of my enemies be equally struck mute against me...”.) 
Dalla Germania, dove questa formula era molto utilizzata anche nelle preghiere per la giustizia, proviene un altro esempio: in esso, chi si lamenta è probabilmente la vedova di Florus, perché è stata derubata da Ulattio Severo della sua proprietà.

(13) Germania, Mogontiacum, DTM 3 (65-130 d. C.):

Rogo te, domina Mater Magna, ut me vindices de bonis Flori coniugis mei, qui me fraudavit Ulattius Severus. Quemadmod(um) hoc ego averse scribo, sic illi omnia, quidquid agit, quidquid aginat, omnia illi aversa fiant, ut sal et aqua illi eveniat. Quidquid mi abstulit de bonis Flori coniugis mei, rogo te domina Mater Magna, ut tu de eo me vindices. ${ }^{18}$

Quomodo, o sporadicamente anche i suoi sinonimi: quemadmodum, ita uti - sic, (tantum quantum), appare allora nella simile formula adoperato per esprimere l'intento di influenzare gli eventi futuri. Tale formula procede sulla base di una analogia, la quale viene espressa tramite il desiderio dell'autore della maledizione, che la vittima possa assomigliare a qualcosa da cui è diversa o che la vittima venga a trovarsi in una situazione, nella quale non si trova in quel momento, usando le completive di comparazione/equazione con le congiunzioni quomodo - sic, ut/ita - sic.

(14) Roma, dfx.1.4.4/3 ( $1^{\circ}$ sec. a. C.):

Quomodo mortuos, qui istic sepultus est nec loqui nec sermonare potest, seic Rhodine apud M(arcum) Licinium Faustum mortua sit nec loqui nec sermonare possit. Ita uti mortuos nec ad deos nec ad homines acceptus est, seic Rhodine aput M(arcum) Licinium accepta sit et tantum valeat, quantum ille mortuos, quei istic sepultus est. Dite pater, Rhodine $(m)$ tibei commendo, uti semper odio sit M(arco) Licinio Fausto... ${ }^{19}$ La frase principale introdotta da sic tematizza l'effetto (desiderato) della maledizione tramite la formula ottativa (wish clause) con congiuntivo volitivo/proibitivo (presente, perfetto): seic Rhodine apud M(arcum) Licinium Faustum mortua sit nec loqui nec sermonare possit...; nella completiva introdotta da quomodo, abitualmente coll'indicativo, si esprime il termine di paragone, cioè a che cosa deve assomigliare la vittima della maledizione: Quomodo mortuos, (qui istic sepultus est) nec loqui nec sermonare potest. Quindi Rhodine non deve essere capace di parlare, così come non ne è capace il morto nel sepolcro. In questa tavoletta vediamo il repertorio quasi completo dei correlativi attestati nelle defixiones; ita $u t i$ - sic appare soltanto in altre due tavolette dalla Germania e dall'Africa. Quemadmodum su altre cinque tavolette. Nei testi delle maledizioni ut come correlativo di quomodo tende a scomparire e resta solo sic, perché $u t$ è impiegato nelle defixiones esclusivamente per introdurre le subordinate finali e consecutive, usate molto frequentemente nelle va-

18 ("I entreat you, Lady Mater Magna, to avenge me regarding the property of Florus, my husband, [of which] Ulattius Severus has defrauded me. Just as I write this in a perverted/hostile way, so may whatever he does and carries out be wasted, may it become salt and water [= salty water]. Whatever he has taken away from me from the property of Florus, my husband, I entreat you, Lady Mater Magna, to avenge me/ exact revenge on him for it.”) Per testo e interpretazione vedi Blänsdorf (2012: No. 3).

19 ("Just like this dead one, who is buried here, cannot speak nor talk [to anyone], may Rhodine be dead for Marcus Licinius Faustus, nor be able to speak or talk [to him]. Just like the dead one is dear neither to gods, nor men, may Rhodine be equally [little] dear to Marcus Licinius, and may she mean to him as much as this dead one who is buried here. Father Dis, I commend to you Rhodine so that she may be always hated by Marcus Licinius Faustus...”.) 
rie formule delle maledizioni; e quindi con sic il significato comparativo/equativo viene ben distinto da quello finale (si veda anche Kropp 2008a: p. 282). Per quanto riguarda l'uso dei modi indicativo e congiuntivo, nella costruzione comparativa/equativa usata nella simile-formula, nei testi delle defixiones di solito nella frase principale si trova usato il congiuntivo, nella completiva con quomodo di solito l'indicativo - come si osserva: quomodo mortuos non potest - sic Rhodine mortua sit, nec sermonare possit; anche nell'altra frase: ita uti mortuos nec acceptus est, sic Rhodine accepta sit... Questo testo presenta sia il congiuntivo perfetto che quello presente, ma di solito viene usato prevalentemente il congiuntivo presente: il congiuntivo perfetto è attestato solo poche volte, in quattro tavolette.

La costruzione tipica per le maledizioni è la combinazione dell'indicativo + il congiuntivo presente nella principale dopo sic, combinazione attestata nella maggioranza dei casi in tutte le province; se ne vede anche il ripetersi tipico delle maledizioni:

(15) Roma, Bevilacqua \& Colacicchi 2009 (2a metà del $1^{\circ}$ sec. d. C.):

Virga, deum inferum ... efficias illae Caeciliae Primae, quem admodum tu domas, caedis uris (per)uris ad Inferos eos, qui ad superos omnia mala sc(el)eraque fecerunt ... sic tu Caeciliam Primam ... uras peruras caedas, domes, do(n)ec, tamqu(a)m al oram egentes omnibus fortunis, mortua $(m)$ ad $t(e)$ abducas... ${ }^{20}$

Mogontiacum, DTM 3, vedi No. (13):

Quemadmod(um) hoc ego averse scribo, sic illi omnia, quidquid agit, quidquid aginat, omnia illi aversa fiant...

Aquincum, Barta 2015, vedi No. (1):

Cuomodo Manes muti et taciti sunt, sic qui tibi antepistulam adferent, muti et taciti sint.

Mogontiacum, DTM 15, vedi No. (9):

... como haec carta nuncquam florescet, sic illa nuncquam florescat.

Rarissimi sono i casi con l'indicativo in ambedue le frasi, probabilmente ascrivibili piuttosto all'errore dello scriba, perché nei testi delle defixiones l'uso del congiuntivo risulta vivo e regolare..$^{21}$ Nell'esempio seguente (16) le divinità infere devono trascinare Aurelio Sinniano agli inferi, affinché sua moglie Silvia lo veda "inverso" - qui probabilmente significa morto.

(16) Noricum, Mautern, dfx.6.1/1 (metà del $2^{\circ}$ sec. d. C.):

Pluton sive Iovem infernum dici opornotet (=oportet), Eracura (=Aeracura) Iuno inferna, acciet $(e)$ ia $(m)$ c(e)lerius infrascribtum e(t) tradite Manibus Aurelium Sinnianum $C(a)$ eserianum. Sic Silvia inversu $(m)$ maritu $(m)$ ceernis (=cernis), quommodi nomen il( $l$ ) ius scriptum est. ${ }^{22}$

20 ("Virga, of the infernal gods ... may you cause ... to that Caecilia Prima. Just as you subjugate, hit, burn, annihilate to Hell those who have committed any crime or impious act towards the gods, so may you, o Virga, burn, annihilate, hit, subjugate that Caecilia Prima until you draw her dead to you, just like those who are devoid of any luck...”.)

[Transl. retrieved 11. 8. 2016 from TheDeMa, Nr. 517, http://www-e.uni-magdeburg.de/defigo/wordpress ].

21 Vedi anche Urbanová \& Cuzzolin (2016: pp. 329-333).

22 ("Oh, Pluto, or if it is necessary to speak to you as Underworld Jupiter, oh, Aeracura, the Underworld Iuno, very quickly summon the above-inscribed Aurelius Sinnianus Caesarianus and hand him over to 
La riduzione della comparativa di modo a sintagma che avviene nel latino letterario (Tarriño 2011: pp. 402-404) come in Petron. 38,15 solebat sic cenare quomodo rex, si verifica anche nei testi delle defixiones e in epigrafi non magiche, ma relativamente di rado, probabilmente perché nelle defixiones tutto ciò che si dice ha anche una sua importanza magica e ridurre una formula potrebbe comportare dei rischi a proposito dell'effetto desiderato.

(17) Gallia, Chagnon, dfx.4.3.1/1 e dfx.4.3.1/2 (metà del 2º sec. d. C.):

... Quomodo hic catellus nemin(i) nocuit, sic ... nec illi hanc litem vincere possint. Quomodi nec mater huius catelli defendere potuit, sic nec advocati eorom e(os d)efendere non possint, sic il(lo)s (in)imicos. (continuazione sul altra tavoletta): aversos (=aversi) ab hac l(i)te esse (debent?). Quomodi hic catellus aversus est nec surgere potesti (=potest), sic nec illi. Sic tra(n)specti (=transfixi) sin(t) quomodi ille. Quomodi in hoc m(o)nimont(o) (=monumento) animalia ommutuerun(t) nec surgere possun $(t)$ nec illi...

Nelle due tavolette di Chagnon è contenuta una maledizione contro i nemici in un processo, in cui la simile formula appare cinque volte, delle quali due completa, due volte ridotta e una volta frammentaria. ${ }^{23}$ Sintagmi ridotti quasi idiomatici sono rari nelle defixiones, un esempio e la tavoletta da Bath, dfx.3.2/1 (vedi punto 3), altre locuzioni sono connesse con piombo e appaiono senza riduzione, come vediamo nel testo di Mogontiacum, DTM 11 (65-130 d. C.): ... Placida et Sacra, filia eius: sic illorum membra liquescan(t) quatmodum hoc plumbum liquescet, ut eoru $(m)$ exsitum sit. ${ }^{24}$

Molto rari sono anche i casi in cui l'ordine delle frasi è invertito con valore espressivo, cosa che non avviene molto di frequente neanche nel corpus letterario: si veda Bodelot (2010: p. 212). In alcuni casi quomodo - sic viene rafforzato con sic all'inizio, mentre una volta appare insieme a $u t$, che enfatizza il desiderio dello scrivente.

Mautern, dfx.6.1/1, vedi No. (16):

Sic Silvia inversu $(m)$ maritu $(m)$ ceernis (=cernis), quommodi nomen il(l)ius scriptum est.

Bad Kreuznach, dfx. 5.1.4/5, No. (10):

Sic comdi plumbum subsidet, sic Sintonem et Martialem Sinto(nis) et adiutorium Sintonis ... defero ad infero(s).

(18) Raetia, Wilten, dfx.7.5/1, vedi anche No. (2) (metà del $2^{\circ}$ sec. d. C.):

Secundina Mercurio et Moltino mandat, ut, si quis *XIIII sive draucus duos sustulit, ut eum sive fortunas eius in $(f i) d u s$ Cacus sic auferat, quomodo ill( a)e ablatum est (i)d, quod vobis delegat, ut persecuatis (=persequatis)... ${ }^{25}$

Manes. May you, Silvia, see your husband inverted, just like his name is written.”). See also Faraone-Kropp (2010: pp. 381-398).

23 Vedi anche Gager (1992: Nr. 53).

24 ("... Placida and Sacra, her daughter: may their limbs melt, just as this lead shall melt, so that it shall be their death.")

25 ("Secundina commends to Mercurius and Moltinus that whoever has stolen two necklaces worth fourteen denarii may be deceived and deprived of property by perfidious Cacus, just as she was deprived of hers, 
In un caso, la simile-formula è attestata in un'altra costruzione con l'imperativo, che ben esprime l'idea o il desiderio dello scrivente, che cosa deve accadere alla vittima. In questo caso nella principale al posto del congiuntivo c'è un imperativo. Il testo della tavoletta, con un layout magico, proviene dall'anfiteatro di Cartagine, ed è purtroppo molto danneggiato; nel centro della tavoletta si trova un rozzo disegno di una figura - demone con le mani incrociate e legate - e proprio ai demoni si riferisce il testo: il demone è legato mediante le formule magiche e costretto a adempiere l'ordine dello scrivente e legare la vittima della maledizione.

(19) Africa, Cartagine dfx.11.1.1/37 (metà del $3^{\circ}$ sec. d. C.):

... q(uomod)o ped(es) (h)abes l(igat)os, sic et eius Salbi (=et eis albis) ligatae (=ligate) pedes Alumno et Pyr(rh)o, Polyarcis et Lascovuo obligatae (=obligate) pedes ... ut obruant. Pre(hendite), (ob)ligatae ped(es) (Ba)tes et Lucife(ro) e(t) (Concordio et Sereno?). ${ }^{26}$

L'uso della simile-formula con quomodo con la frase ottativa e congiuntivo volitivo/proibitivo dopo sic è attestato rarissimamente anche nelle epigrafi communi, ad es. su un epitaffio da Roma:

(20) AE 1976, 94, databile tra 50 a. C. - 30 d. C.:

Quisque hui(c) loco bene / fecerit sic senescat quomodo / ego sic audiat quo modo ego / fecerunt conliberti Maiori / suo bene merenti.

Oppure in un graffito da Pompei con imperativo:

(21) CLE 01798a:

Labora aselle, quomodo ego laboravi, et proderit tibi. ${ }^{27}$

Nelle epigrafi non troppo frammentarie, in tutte le altre occorrenze delle completive con quomodo - sic nelle epigrafi communi si usano indicativi (vedi ad es. CLE 01542, Didymoi 00429).

La costruzione di quomodo con congiuntivo nella principale non è però attestata nel latino letterario, né menzionata dalle grammatiche latine. Nel latino letterario si usa di solito l'indicativo (Hofmann \& Szantyr 1965: p. 648) e altri, mentre il congiuntivo compare soltanto in pochi casi nell'oratio obliqua. Nel Brepols Cross Database Searchtool, ${ }^{28}$ ho trovato soltanto due esempi assomiglianti all'uso documentato dalle defixiones, il primo con indicativo, il secondo, tratto da Seneca filosofo, con congiuntivo obliquo.

(22) Epistularium Frontonis - M. Antonini imperatoris epistulae ad Frontonem, liber: 1, epist.: 4, par.: 1: Oro te, mi magister, ama me ut amas; ama me sic etiam, quomodo istos parvolos nostros amas; nondum omne dixi quod volo: ama me quomodo amasti.

which she orders you to trace back...”). This is the interpretation of J. G. Gager (1992: No. 101) who associates the word draucus with draukion, a Greek term for necklace.

26 ("... Just as your feet are tied up, may you [in plural] bind the hooves of [the horses of white team?] Alumnus and Pyrus, bind up the hooves of Polyarcus and Lascivius ... so that they fall over. Seize, bind up the hooves of Bates and Lucifer and...”.)

27 Vedi anche Tarriño (2011: p. 406).

28 Brepols Publishers, Turnhout, 2015. [Retrieved 11. 8. 2016 from http://www.brepolis.net]. 
(23) Seneca, De beneficiis, lib.: 2, cap.: 1, par.: 1: Inspiciamus, liberalis virorum optime, id quod ex priore parte adhuc superest, quemadmodum dandum sit beneficium; cuius rei expeditissimam videor monstraturus viam: sic demus, quomodo vellemus accipere.

A questo punto sorge la questione: da dove proviene la costruzione quomodo - sic più congiuntivo nell'ambito delle defixiones? Perché non è attestata nella lingua letteraria, quindi fuori dell'ambito delle maledizioni? Non è nemmeno chiaro se nelle defixiones latine si tratti di uno sviluppo indipendente dalla lingua parlata o scritta latina a causa del contesto magico, o se sia un prestito dal linguaggio magico greco impiegato nelle maledizioni.

Anche nelle tavolette greche si trova l'uso di tale simile-formula, attestato già dal quar-

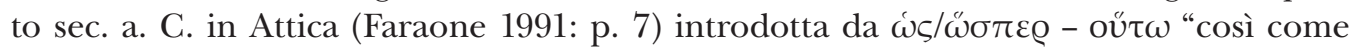
- così", la quale corrisponde al latino quomodo - sic. Kagarow (1929: pp. 32-34) distingue questa formula in greco o con imperativo o con ottativo.

(24) DT No. 241 (15-18), con imperativo:

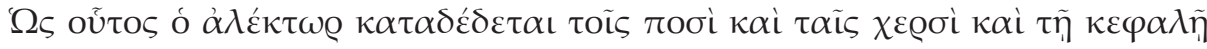

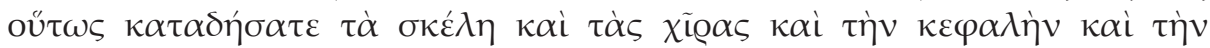

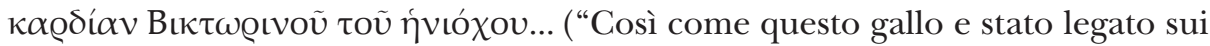
piedi e mani (ali) e sulla testa, così legate le gambe, le mani, la testa e il cuore dell'auriga Victorino...”).

(25) Wünsch (1897: No. 67a):

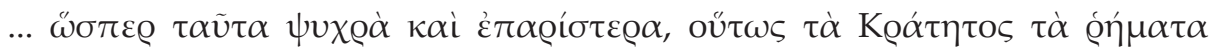

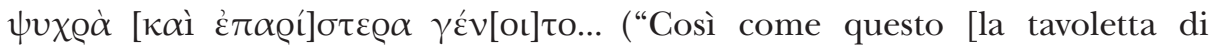
piombo] è freddo e scritto sinistrorso, così anche le parole di Krates siano fredde e sinistrorse"), la tavoletta è scritta con ductus sinistrorso.

A questo punto ora non si può dire con sicurezza né se o in quale modo l'uso "magico" di quomodo ne ha orientato lo sviluppo nella storia di lingua latina e transizione alle lingue romanze, e nemmeno se quomodo nelle maledizioni sia un calco dal greco, che è un ipotesi che sembra plausibile e anzi molto probabile, o se si tratti di un sviluppo proprio e indipendente nella lingua latina. Tutto ciò resta ancora da esaminare. ${ }^{29}$

\section{Abbreviazioni:}

AE L'Année Épigraphique

CLE Buecheler, F. (Ed.). (1895). Carmina Latina Epigraphica (Fasc. I). Lipsiae: Teubner.

dfx. $\quad$ Kropp, A. (2008). Defixiones. Ein aktuelles Corpus lateinischer Fluchtafeln. Speyer: Kartoffeldruck-Verlag Kai Brodersen.

Didymoi Cuvigny, H. (2012). Didymoi. Une garnison romaine dans le désert oriental d'Égypte, Band 2: Les textes. Kairo: Institut Français d'Archéologie Orientale.

DT Audollent, A. (1904). Defixionum tabellae. Paris: Albert Fontemoing.

29 Sono molto grata a Pierluigi Cuzzolin per vari suggerimenti nella revisione del testo italiano e a Natália Gachallová per le traduzioni dei testi delle defixiones in inglese. 
DTM Blänsdorf, J. (2012). Die defixionum tabellae des Mainzer Isis- und Mater Magna-Heiligtums, Defixionum tabellae Mogontiacenses (DTM) (in Zusammenarbeit mit Pierre-Yves Lambert und Marion Witteyer). Mainz: Generaldirektion Kulturelles Erbe Rheinland-Pfalz, Direktion Landesarchäologie.

EDCS Epigraphik-Datenbank Clauss/Slaby [http://db.edcs.eu/epigr/epi_de.php; accessed 11. 8. 2016].

RIB Roman Inscriptions of Britain [http://romaninscriptionsofbritain.org/inscriptions/154; accessed 11. 8. 2016].

TheDeMa Thesaurus Defixionum Magdeburgensis

[http://www-e.uni-magdeburg.de/defigo/wordpress; accessed 11. 8. 2016].

\section{Bibliografia}

Adams, J. (1997). The Vulgar Latin of the Letters of Claudius Terentianus (P. Mich. VIII, 467-72). Manchester: Manchester University Press.

Adams, J. (2007). The Regional Diversification of Latin, 200 BC - 600 AD. Cambridge: Cambridge University Press.

Barta, A. (2009). The Language of Latin Curse Tablets from Pannonia. A New Curse Tablet from Aquincum. Acta Antiqua Academiae Scientiarum Hungaricae, 49, 23-29.

Barta, A. (2012). Milites magistratusque. A new curse tablet from Savaria. Acta classica Universitatis Scientiarum Debreceniensis, 48, 167-173.

Barta, A. (2015). Ito pater, Eracura and the Messenger. A Preliminary Report on a New Curse Tablet from Aquincum. Acta Classica Universitatis Scientiarum Debreceniensis, 51, 101-103.

Barta, A. (2016). A Letter to the Underworld. A Research Report on the Curse Tablet Aq-2. Acta Antiqua Academiae Scientiarum Hungaricae, 56 (in stampa).

Bevilaqua, G., \& Colacicchi, O. (2009). Una nuova defixio latina dalla via Ostiense. Notizie degli scavi di antichità, Ser. IX, Vol. XVII-XVIII, 2006-2007, 303-349.

Blänsdorf, J. (2014). Das Verfluchungstäfelchen aus Gelduba (Gellep bei Krefeld) Grab 5486. Zeitschrift für Papyrologie und Epigraphik, 92, 181-186.

Blänsdorf, J., Kropp, A., \& Scholz, M. (2010). ,Perverse agas, comodo hoc perverse scriptu(m) est - ein Fluchtäfelchen aus Köln. Zeitschrift für Papyrologie und Epigraphik, 174, 272-276.

Blänsdorf, J., \& Piranomonte, M. (2012). Schede di catalogo IX, 49.3.-6, IX, 49.8-28. La fontana di Anna Perenna. In R. Friggeri, M. G. Granino Cecere, \& G. L. Gregori (Eds.), Terme di Diocleziano. La collezione epigrafica (pp. 617-639). Milano: Electa.

Bodelot, C. (2010). Quo(=)modo relatif / comparatif: son évolution du latin préclassique au latin postclassique. In P. Peter Anreiter, \& M. Kienpointner (Eds.), Latin Linguistics Today. Akten des 15. Internationalen Kolloquiums zur lateinischen Linguistik (Vol. 137; pp. 209-220). Innsbruck: Innsbrucker Beiträge zur Sprachwissenschaft.

Bodelot, C. (2012). Les emplois de quomodo chez Lucifer de Cagliari. In F. Biville, M.-K. Lhommé, \& D. Vallat (Eds.), Latin vulgaire - Latin tardif, IX: Actes du IX colloque international sur le latin vulgaire et tardif (pp. 425-437). Lyon: Maison de l'Orient et de la Méditerranée - Jean Pouiloux. Cuzzolin, P. (2011). Comparative and superlative. In Ph. Baldi, \& P. Cuzzolin (Eds.), New Perspec- 
tives on Historical Latin Syntax, 4: Complex Sentences, Grammaticalization, Typology (pp. 549-660). Boston-Berlin: Walter de Gruyter.

Faraone, Ch. A. (1991). The Agonistic Context of Early Greek Binding Spells. In Ch. A. Faraone, \& D. Obbink (Eds.), Magika Hiera. Ancient Greek Magic and Religion (pp. 3-32). Oxford: Oxford University Press.

Faraone, Ch. A., \& Kropp, A. (2010). Inversion, Adversion and Perversion as Strategies in Latin Curse-Tablets“. In R. L. Gordon, \& F. M. Simón (Eds.), Magical Practice in the Latin West. (pp. 381-398). Leiden: Brill.

Fontana Elboj, G. (2002). Las construcciones comparativas latinas: estado de la cuestión. In E. Espinilla, P. J. Quetglas, \& M. E. Torrego (Eds.), La comparación en latín (pp. 81-104). Madrid-Barcelona: Universidad Autónoma de Madrid, Universitat de Barcelona.

Gager, J. G. (1992). Curse Tablets and Binding Spells from the Ancient World. Oxford: Oxford University Press.

Haspelmat, M., \& Buchholz, O. (1998). Equative and similative constructions in the languages of Europe. In J. van der Auwera in collsb. with D. Ó Baoill, (Eds.), Adverbial Constructions in the Languages of Europe (pp. 277-334). De Gruyter Mouton: Berlin.

Herman, J. (1957). Cur, quare, quomodo, remarques sur l'évolution des particules d'interrogation en latin vulgaire. Acta Antiqua Academiae Scientiarum Hungaricae, 5(1-4), 370-377.

Herman, J. (2000) [1967]. Vulgar Latin (Engl. transl. by R. Wright). University Park, Pa: Pennsylvania State University Press.

Hoffmann, J. B., \& Szantyr, A. (1965). Lateinische Syntax und Stylistik. München: Beck.

Kagarow, E. (1929). Griechische Fluchtafeln (Eos Supplementa, 4). Paris: Les Belles Lettres.

Kropp, A. (2008a). Magische Sprachverwendung in vulgärlateinischen Fluchtafeln. Tübingen: Gunter Narr Verlag.

Kropp, A. (2010). How does Magical Language Work? The Spells and Formulae of the Latin Defixionum Tabellae. In R. L. Gordon, \& F. M. Simón (Eds.), Magical Practice in the Latin West (pp. 357-380). Leiden: Brill.

Menge, H. (2000). Lehrbuch der lateinischen Syntax und Semantik (neu bearb. Th. Burkard und M. Schauer). Darmstadt: Wissenschaftliche Buchgesellschaft.

Morani, M. (2000). Introduzione alla linguistica latina. München: Lincom Europa.

Pirson, J. (1908). Quomodo en latin vulgaire. In K. Reuscher, \& K. Gruber (Eds.), Philologische und volkskundliche Arbeiten Karl Vollmöller zum 16. Oktober 1908 dargeboten von Gottfried Beist (pp. 61-74). Erlangen: Verlag Fr. Junge.

Revuelta Puigdollers, A. (2002). Oraciones comparativas de igualidad: nivelles de integración. In E. Espinilla, P. J. Quetglas, \& M. E. Torrego (Eds.), La comparación en latín (pp. 191-228). Madrid-Barcelona: Universidad Autónoma de Madrid-Universitat de Barcelona.

Tarriño, E. (2011). Comparative clauses. Comparative and superlative. In Ph. Baldi, \& P. Cuzzolin (Eds.), New Perspectives on Historical Latin Syntax, 4: Complex Sentences, Grammaticalization, Typology (pp. 373-426). Boston-Berlin: Walter de Gruyter.

Tomlin, R. S. O. (1988). The Curse Tablets. In B. Cunliffe (Ed.), The Temple of Sulis Minerva at Bath, 2: The Finds from the Sacred Spring (pp. 59-270). Oxford: Oxford University Cometee for Archaeology. 
Tomlin, R. S. O. (1993). The Inscribed Lead Tablets: An Interim Report. In A. Woodward, \& P. Leach (Eds.), The Uley Shrines. Excavation of a Ritual Complex on West Hill, Uley, Gloucestershire 1977-1979 (pp. 112-130). London: English Heritage.

Tomlin, R. S. O, \& Hassall, M. W. C. (1989). II Inscriptions. Britannia, 20, 329-330.

Torrego, M. E. (2002). Los SN comparativos: el segundo término de la comparación. In E. Espinilla, P. J. Quetglas, \& M. E. Torrego (Eds.), La comparación en latín (pp. 251-279). Madrid-Barcelona: Universidad Autónoma de Madrid, Universitat de Barcelona.

Urbanová, D. (2014). Die lateinischen tabellae defixionum, der Usus und die Spezifika am Gebiet des römischen Reiches. In P. Molinelli, P. Cuzzolin, \& Ch. Fedriani (Eds.), Latin vulgaire - Latin tardif, X: Actes du Xe colloque international sur le latin vulgaire et tardif (Vol. III; pp. 1070-1081). Bergamo: Bergamo University Press, Sestante edizioni.

Urbanová, D. (2014a). Latinské proklinaci tabulky na územi Řimského impéria. Brno: Host.

Urbanová, D., \& Cuzzolin, P. (2016). Some linguistic and pragmatic remarks on the tabellae defixionum. Journal of Latin Linguistics 15(2), 313-345.

Väänänen, V. (1951). Il est venu comme ambassadeur, il agit en soldat. Et locutions analogues en latin, français, italien et espagnol. Annales Academiae Scientiarum Fennicae, Ser. B, 73(1), 1-74.

Väänänen, V. (1982) [1963]. Introduzione al latino vulgare (Transl. A. G. Silvestri). Bologna: Pàtron editore.

Wünsch, R. (1897). Defixionum tabellae Atticae. Berolini: Reimer.

Doc. PhDr. Daniela Urbanová, Ph.D. / urbanova@phil.muni.cz

Department of Classical Studies

Masaryk University, Faculty of Arts

Arna Nováka 1, 60200 Brno, Czech Republic 
